\title{
Enhancement of Effective Accumulation of Atoms during Fabrication of Al Microsphere
}

\author{
Faizul Mohammad Kamal*, Md. Nazrul Islam Khan, Sheikh Manjura Hoque \\ Materials Science Division, Atomic Energy Centre, Dhaka, Bangladesh \\ Email: ^kamal_ndt@yahoo.com
}

How to cite this paper: Kamal, F.M., Khan, Md.N.I. and Hoque, S.M. (2019) Enhancement of Effective Accumulation of Atoms during Fabrication of Al Microsphere. Microscopy Research, 7, 39-46. https://doi.org/10.4236/mr.2019.74004

Received: July 12, 2019

Accepted: October 28, 2019

Published: October 31, 2019

Copyright ( 2019 by author(s) and Scientific Research Publishing Inc. This work is licensed under the Creative Commons Attribution International License (CC BY 4.0).

http://creativecommons.org/licenses/by/4.0/

\begin{abstract}
Microspheres of $\mathrm{Al}$ have been successfully fabricated utilizing electromigration using sudden change in geometrical shape of a specimen. The experimental sample was a passivated $\mathrm{Al}$ line with a hole at the transitional area of the sample. The hole was used to control the accumulation and discharge process. The formation of the microsphere is enhanced by controlling temperature and current density. The atomic flux was increased with the increasing current density that was happened along the electron flow direction in the small region at the geometrical shape of the sample.
\end{abstract}

\section{Keywords}

Electromigration, Atoms, Migration, Microsphere, Accumulation

\section{Introduction}

Elecrtromigration (EM) is an atomic migration phenomenon wherein metallic atoms are transported by electron wind due to high current density in a metal line. EM produces atomic accumulations known as hillocks and vacancies as voids that cause short or open circuits. EM is very important techniques for fabricating $\mathrm{Al}$ micro and nano materials (MNMs). In addition, different elemental materials have been manufactured by chemical [1] [2] [3] [4] and physical approaches [5] [6] [7] [8]. However, Al MNMs are difficult to fabricate because of their high reactivity to acids and bases. Nevertheless, various approaches for fabricating $\mathrm{Al}$ MNMs have been developed by physical approaches. Various MNMs such as micro-wire [9], micro-belt [10], micro-tube [11] have been successfully fabricated by introducing an artificial slit at the anode end of an $\mathrm{Al}$ line to promote atomic accumulation. Fabrication of an $\mathrm{Al}$ microsphere using EM methods by introducing an artificial slit at the anode end of the sample has previously been demonstrated [12]. However, the introduction of a slit is quite time-con- 
suming and also makes this fabrication method costly. Therefore, a hint at a possible breakthrough is provided by latest findings [13], which indicate that an $\mathrm{Al}$ line structure that undergoes a sudden change in geometrical shape can effectively promote the accumulation of atoms during EM.

In the present research, the sudden change in the geometrical shape of the metal specimen could be expected to enhance accumulation of atoms. Therefore, a technique of fabricating of microsphere has been proposed by EM using sudden change in geometrical shape of the sample. In addition, the effectiveness of this technique was verified with optimization of high temperature and current density by formation of microsphere from a predefined hole.

\section{Experimental Procedure}

A schematic illustration of the sample structure used in the present work is shown in Figure 1(a). The sample fabrication process is summarized as follows: First, a Si [100] substrate with a thickness of $290 \mu \mathrm{m}$ was thermally oxidized to form a $0.3-\mu \mathrm{m}$-thick $\mathrm{SiO}_{2}$ layer on the surface. Then, a $0.3-\mu \mathrm{m}$-thick titanium nitride (TiN) layer was sputtered onto the $\mathrm{SiO}_{2}$ layer; this TiN plays several important roles, including preventing the atomic migration of $\mathrm{Al}$ toward the $\mathrm{Si}$ wafer, promoting adhesion between $\mathrm{Al}$ and the $\mathrm{Si}$ wafer, and providing a bypass for current in case an electrical shortcut is induced by the growth of voids due to EM. Subsequently, a $0.6-\mu \mathrm{m}$-thick $\mathrm{Al}$ film was deposited on the TiN layer by vacuum evaporation using an $\mathrm{Al}$ source of $99 \%$ purity $(2 \mathrm{~N})$. The $\mathrm{Al}$ and TiN layers were then patterned by wet etching and fast atom beam (FAB) etching, respectively, to obtain an ideal sample structure with a sudden change in geometrical shape as shown in Figure 1. The narrow part of the $\mathrm{Al}$ line has a width of $12 \mu \mathrm{m}$, and the wide part of the $\mathrm{Al}$ line has a width of $424 \mu \mathrm{m}$. The Al sample has the length of $100 \mu \mathrm{m}$. Then, a $2.4-\mu \mathrm{m} \mathrm{SiO}{ }_{2}$ film was deposited over the $\mathrm{Al}$ surface by plasma-enhanced chemical vapor deposition (PE-CVD); this film acted as a passivation layer to provide sufficient compressive stress. Finally, a hole with a diameter of $1 \mu \mathrm{m}$ was etched by focused ion beam (FIB) to control the discharge of accumulated $\mathrm{Al}$ atoms. Figure 1 (b) shows the cross-sectional view of the sample.

The sample was placed on a ceramic heater under atmospheric conditions, and a substrate temperature was maintained at $623 \mathrm{~K}$. The samples were then subjected to a direct current flow using a pair of probes in contact with the input and output pads. Figure 2(a) shows the experimental sample with sudden change in geometrical shape. During the EM experiment, substrate temperature, $T_{\mathrm{s}}=623 \mathrm{~K}$ and current density, $|\mathrm{j}|=8.33 \mathrm{MA} / \mathrm{cm}^{2}$ was optimized for accumulation of atoms at the sudden change in geometrical shape of metal line.

\section{Results and Discussion}

The experimental conditions and results are summarized in Table 1. During the EM experiment, substrate temperature, $T_{s}=623 \mathrm{~K}$ and current density, $|j|=8.33$ $\mathrm{MA} / \mathrm{cm}^{2}$ was applied for accumulation of atoms at the sudden change in geometrical shape of metal line. Image of typical feature after the passage of current, 
obtained by field emission scanning electron microscope (FE-SEM), is shown in Figure 2. Microspheres were fabricated at the predefined location in the sample with a sudden change in geometrical shape, which is shown in Figure 2(b). Here, four specimens were used under the same electrical conditions.

Table 1. Test conditions used in experiments.

\begin{tabular}{|c|c|c|c|c|c|}
\hline Sample & $\begin{array}{c}\text { Substrate } \\
\text { temperature, } \\
T_{s}(\mathrm{~K})\end{array}$ & $\begin{array}{c}\text { Current density, } \\
j \\
\left(\mathrm{MA} / \mathrm{cm}^{2}\right)\end{array}$ & $\begin{array}{c}\text { Current } \\
\text { stressing time } \\
\text { (s) }\end{array}$ & $\begin{array}{l}\text { Formed } \\
\text { Structure }\end{array}$ & $\begin{array}{c}\text { Diameter } \\
\text { of sphere } \\
(\mu \mathrm{m})\end{array}$ \\
\hline S1 & 623 & 8.33 & 1860 & Microsphere & 6.54 \\
\hline S2 & 623 & 8.33 & 1920 & $\begin{array}{l}\text { Microsphere } \\
\text { goes outside }\end{array}$ & 7.48 \\
\hline $\mathrm{S} 3$ & 623 & 8.33 & 1320 & Microsphere & 7.46 \\
\hline S4 & 623 & 8.33 & 1620 & $\begin{array}{l}\text { Microsphere } \\
\text { goes outside }\end{array}$ & 8.41 \\
\hline
\end{tabular}

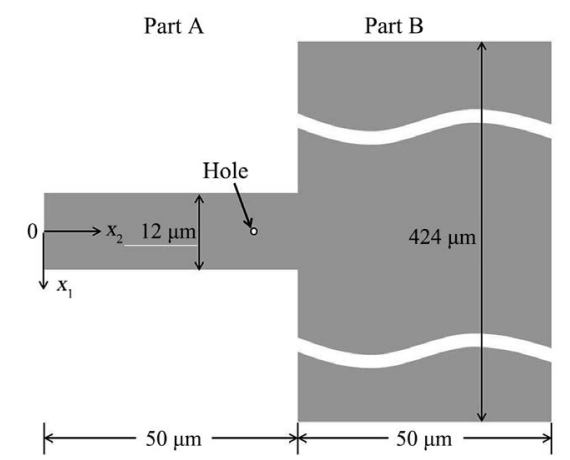

(a)

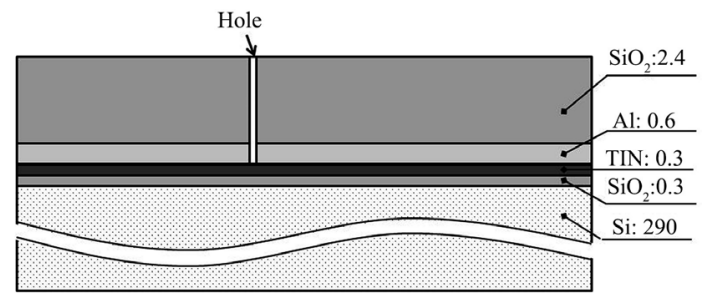

(b)

Figure 1. Schematic of (a) sample structure, and (b) cross-sectional view of the sample.

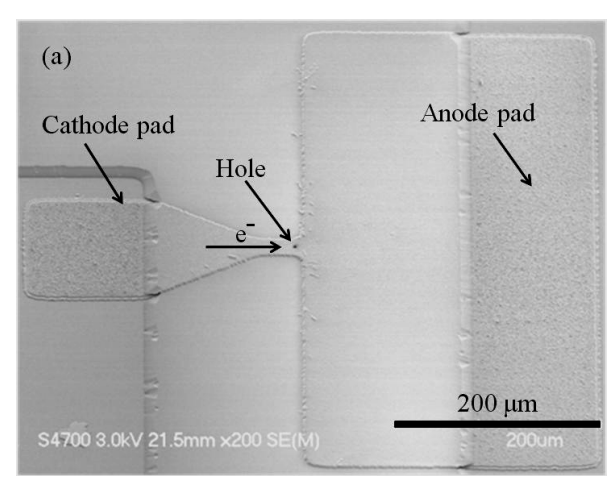

(a) 


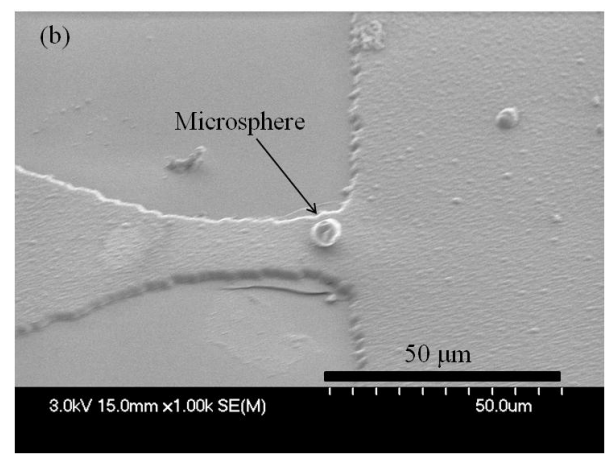

(b)

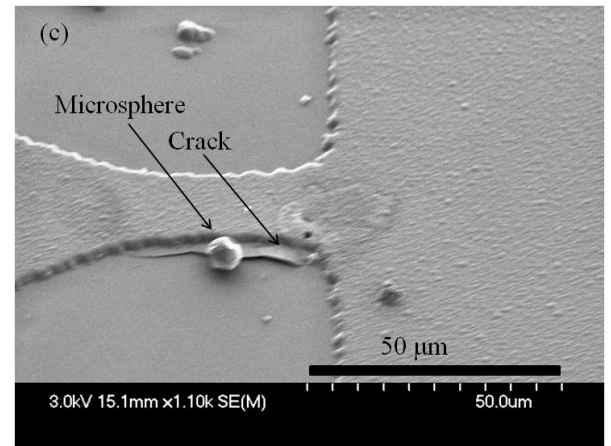

(c)

Figure 2. FE-SEM images of the sample with a sudden change in geometrical shape. (a) Experimental sample with a hole at specific position. (b) Microsphere and cracks formed in sample S1. (c) Large cracks on the single side and microsphere becomes outside of sample in sample S2.

Al microsphere was successfully fabricated which denoted as sample S1 in Table 1. In this case, microsphere with a diameter of $6.54 \mu \mathrm{m}$ was happened at the sudden change in geometrical shape of metal line by supplying a constant substrate temperature, $T_{\mathrm{s}}=623 \mathrm{~K}$ and current density, $|\mathrm{j}|=8.33 \mathrm{MA} / \mathrm{cm}^{2}$, which has been optimized during the experiment and is shown in Figure 2(b). The current stressing time over the experiment was 1860s. Here four specimens with a sudden change in geometrical shape were used under the same current density and substrate temperature of the experiment. In addition, crack and microsphere formed outside of the sample was also observed as sample S2 in Table 1, as shown in Figure 2(c). Therefore, formation of microspheres were observed at the hole location in two specimens, which indicates to some extent the experimental repeatability. In contrast, microspheres go outside and cracks were observed at the $\mathrm{SiO}_{2}$ /substrate interface adjacent to the $\mathrm{Al}$ line in the two specimens. The test conditions and diameter of the microspheres and microspheres goes outside of the samples were also shown in Table 1. The current stressing conditions are quite different between these samples, in which similar condition of higher temperature and higher current densities were observed for the present sample structure.

Electromigration (EM) is a physical phenomenon wherein atoms are trans- 
ported by an electron wind. The number of atoms are transported depends on the current density, temperature and the structure of the metal line [14] [15]. The electron wind due to high current density can drive metallic atoms to a specific location in a structure. In the present study, the effective accumulation of atoms happened at the specific location of the sudden change in the width of the metal line, leading to fabrication of the desired microsphere.

Actually, atomic flux along the electron flow direction in the small region around the predefined hole could be very important to enhance the accumulation of atoms in the $\mathrm{Al}$ line with the sudden change in geometrical shape. The atomic flux due to EM, J, can be described from Huntington and Grone equation [1]:

$$
J=\frac{N D_{0}}{k T} \exp \left(-\frac{Q}{k T}\right) Z * e \rho j
$$

where, $N$ is the atomic density, $D_{0}$ is the pre factor, $k$ the Boltzmann constant, $T$ stands for the absolute temperature, $Q$ represents the activation energy, $Z^{\star}$ is the effective valence, $e$ is the electronic charge, $\rho$ is the electrical resistivity and the symbol $j$ represents the current density.

The temperature and current density distribution of the $\mathrm{Al}$ line were analyzed by two dimensional finite element analysis (FEA). Therefore, FEA was used to explain the experimental results by solving the electro-thermal problem using the software program MSC. Marc/Mentat. The temperature and current density distribution along the $\mathrm{x}_{2}$-axis of the sample with sudden change in geometrical shape are shown in Figure 3. The temperature distribution along the metal line that absolute value of temperature gradient is higher in part A than that of part $B$ of the sample as shown in Figure 3(a). Due to high temperature, more atoms were diffused in part A as small region. On the other hand, higher current density continues up to part A and therefore, sudden decrease in current density happens near the geometrical transition of the sample along the electron flow direction, which is shown in Figure 3(b). In the relationship between atomic flux, $J$ and the temperature $T$ is more complicated, and it also depends on several parameters, especially the sample structure with sudden change in geometrical shape and the value of activation energy, $Q$. Therefore, it is observed that with any specified $Q$ for either grain boundary diffusion or lattice diffusion, $J$ in the present sample structure within the corresponding limited temperature range, increases initially with the increasing $T$ monolithically and then decreases rapidly. However, as given in Equation (1), the atomic flux, $J$ increases with the increasing current density, $j$ monolithically along the electron flow direction in the small region on the sample with sudden change in geometrical shape. Therefore, atomic flux is higher in Part A than that at Part B according to Equation (1). Thus, large number of atoms enters the small region than flow out. Therefore, more atoms are accumulated in the Part A as small region, near the geometrical transition, and atoms are discharged through the hole. Finally, formation of microspheres was happened. 


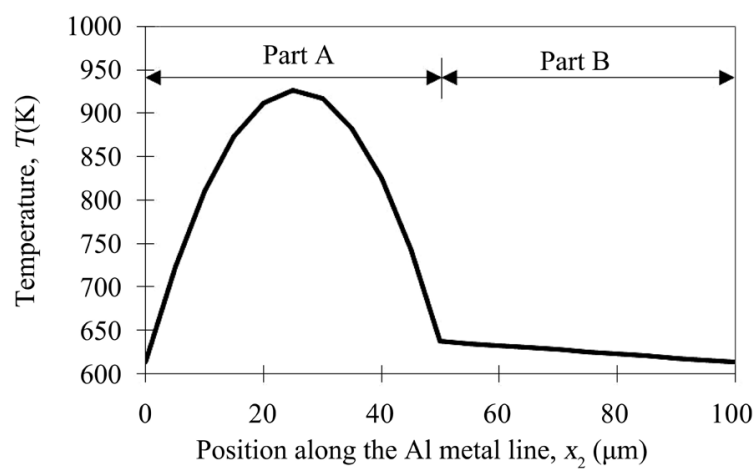

(a)

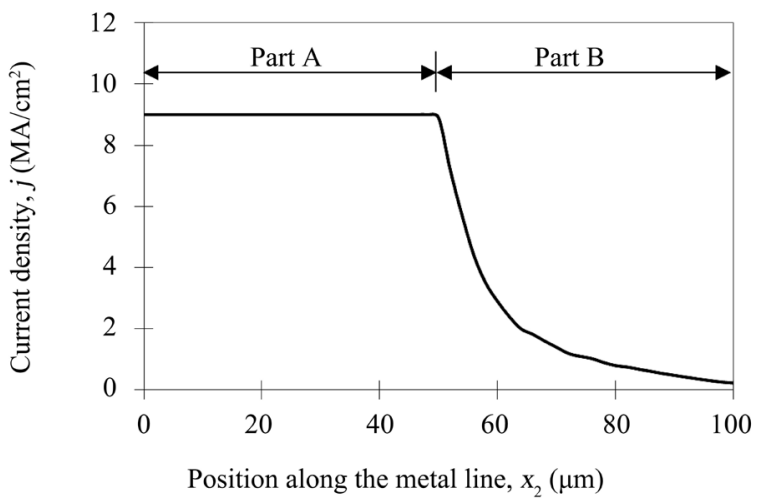

(b)

Figure 3. 2D-FEA results of sample. (a) Temperature distribution. (b) Current density distribution.

The maximum temperature and current density have been occurred near the geometrical transitional area of the present sample with sudden change in geometrical shape. Therefore, the temperature distribution has been happened along the metal line. Finally, the present sample structure using sudden change in geometrical shape of atoms provides the successful formation of microspheres. However, it can be successfully control the accumulation and discharge of atoms to form microspheres.

\section{Conclusion}

An approach for fabrication of microspheres with Aluminum specimen has been attempted in this experiment. Al atoms were accumulated at the geometrical shape in the metal line by electromigration and discharge of atoms was happened in the hole of the sample. Finally, microspheres were formed by optimizing both of the temperature and current density at the predefined location near the geometrical transition of sample. FEA was also used to explain the experimental results by solving the electro-thermal problem on the sample with sudden change in geometrical shape.

\section{Acknowledgements}

Part of the experimental work was carried out at the Micro/Nano-Machining 
Research and Education Centre of Tohoku University. The simulation was performed using supercomputing resources at the Cyberscience Center of Tohoku University.

\section{Conflicts of Interest}

The authors declare no conflicts of interest regarding the publication of this paper.

\section{References}

[1] Tian, M., Wang, J., Snyder, J., Kurtz, J., Liu, Y., Schiffer, P., Mallouk, T.E. and Chan, M.H.W. (2003) Synthesis and Characterization of Superconducting Single-Crystal Sn Nanowires. Applied Physics Letter, 83, 1620-1622. https://doi.org/10.1063/1.1601692

[2] Motoyama, M., Fukunaka, Y., Sakka, T., Ogata, Y.H. and Kikuchi, S. (2005) Electrochemical Processing of $\mathrm{Cu}$ and Ni Nanowire Arrays. Journal of Electroanalytical Chemistry, 584, 84-91. https://doi.org/10.1016/j.jelechem.2005.07.023

[3] Brenner, S.S. (1956) The Growth of Whiskers by the Reduction of Metal Salts. Acta Metallurgica, 4, 62-74. https://doi.org/10.1016/0001-6160(56)90111-0

[4] Zhang, J., Qing, X., Jiang, F. and Dai, Z. (2003) A Route to Ag-Catalyzed Growth of the Semiconducting $\operatorname{In}_{2} \mathrm{O}_{3}$ Nanowires. Chemical Physics Letters, 371, 311-316. https://doi.org/10.1016/S0009-2614(03)00272-0

[5] Black, J.R. (1969) Electromigration-A Brief Survey and Some Recent Results. IEEE Transactions on Electronic Devices, 16, 338-347. https://doi.org/10.1109/T-ED.1969.16754

[6] Blech, I.A. and Herring, C. (1976) Stress Generation by Electromigration. Applied Physics Letters, 29, 131-133. https://doi.org/10.1063/1.89024

[7] Korhonen, M.A., Borgesen, P., Tu, K.N. and Li, C.Y. (1993) Stress Evolution Due to Electromigration in Confined Metal Lines. Journal of Applied Physics, 73, 3790 3799. https://doi.org/10.1063/1.354073

[8] Saka, M., Yamaya, M. and Tohmyoh, H. (2007) Rapid and Mass Growth of StressInduced Nanowhiskers on the Surfaces of Evaporated Polycrystalline Cu Films. Scripta Materialia, 56, 1031-1034. https://doi.org/10.1016/j.scriptamat.2007.02.036

[9] Saka, M. and Nakanishi, R. (2006) Formation of Al Thin Wire by Utilizing Controlled Accumulation of Atoms Due to Electromigration. Materials Letters, 60, 2129-2131. https://doi.org/10.1016/j.matlet.2005.12.107

[10] Lu, Y. and Saka, M. (2009) Fabrication of Al Micro-Belts by Utilizing Electromigration. Materials Letters, 63, 2227-2229. https://doi.org/10.1016/j.matlet.2009.07.031

[11] Lu, Y., Tohmyoh, H. and Saka, M. (2011) Forming Microstructures by Controlling the Accumulation and Discharge of Al Atoms by Electromigration. Journal of Physics D: Applied Physics, 44, Article ID: 045501. https://doi.org/10.1088/0022-3727/44/4/045501

[12] Sun, Y., Tohmyoh, H. and Saka, M. (2009) Fabrication of Al Microspheres by Utilizing Electromigration. Journal of Nanoscience and Nanotechnology, 9, 1972-1975. https://doi.org/10.1166/jnn.2009.444

[13] Kamal, F.M. and Saka, M. (2011) An Approach Using Sudden Change in Geometrical Shape of a Sample for Fabricating Micro/Nano Metallic Materials Utilizing Elctromigration. Proceedings of 13 th International Conference on Electronic Mate- 
rials and Packaging (EMAP2011), Kyoto, 12-15 December 2011.

[14] Huntington, H.B. and Grone, A.R. (1961) Current Induced Marker Motion in Gold Wires. Journal of Physics and Chemistry of Solids, 20, 76-87. https://doi.org/10.1016/0022-3697(61)90138-X

[15] Blech, A. (1976) Electromigration in Thin Aluminum Films on Titanium Nitride. Journal of Applied Physics, 47, 1203-1208. https://doi.org/10.1063/1.322842 